\title{
A Time-Varying Gain Design Method for State Feedback Control of Upper Triangular Nonlinear Systems
}

\author{
Yanmin Yin \\ School of Science, Shandong Jianzhu University, Jinan 250101, China \\ Correspondence should be addressed to Yanmin Yin; yym@sdjzu.edu.cn
}

Received 19 March 2020; Accepted 17 April 2020; Published 5 May 2020

Guest Editor: Rongwei Guo

Copyright (c) 2020 Yanmin Yin. This is an open access article distributed under the Creative Commons Attribution License, which permits unrestricted use, distribution, and reproduction in any medium, provided the original work is properly cited.

\begin{abstract}
In this paper, a time-varying gain design method is used to investigate the state feedback control problem of upper triangular nonlinear systems. Firstly, the nonlinear term recognizes an incremental rate relying on the unknown constant and the function with respect to time. Then, a time-varying gain design method is utilized to construct a state feedback controller. With the help of a suitable coordinate transformation and a Lyapunov function, one obtains that all the signals of the closed-loop system converge to zero. Finally, two numerical examples are presented to display the effectiveness of the time-varying gain design method.
\end{abstract}

\section{Introduction}

Many physical models can be described by nonlinear systems [1-4]. Therefore, the control problem of these physical models can be transformed into the control problem of nonlinear systems [5]. Compared with linear systems, the behavior of nonlinear systems is more diverse [6-10]. The research of control algorithms is generally developed for the specific type of nonlinear systems $[11,12]$.

In general, many results about nonlinear systems have focused on nonlinear systems with triangular structures, that is, lower triangular nonlinear systems $[13,14]$ and upper triangular nonlinear systems [15]. The common method for studying lower triangular nonlinear systems is the backstepping design method [16], and the common method for considering upper triangular nonlinear systems is the forwarding design method [17]. Although, based on the iterative design algorithm, these methods can effectively deal with strong nonlinearities, the design procedure is more complicated. In the past few decades, the gain design method is a very effective tool to deal with the control problem of upper triangular nonlinear systems [18].

Based on the coordinate transformation, the timevarying gain design method is an effective strategy for dealing with the uncertain parameter of upper triangular nonlinear systems [19]. By introducing a time-varying function in the controller, it can effectively deal with the nonlinear terms of upper triangular nonlinear systems [20]. Compared with the commonly adaptive control strategy, the time-varying gain design method is more concise, the calculation process is less, and a lot of calculation work is reduced. Furthermore, the time-varying gain design method does not require too many design parameters and avoids complicated calculation process.

This paper uses the time-varying gain design method to study the control problem of upper triangular nonlinear systems. The nonlinear characteristics of the system considering here are more obvious, that is, the unknown constant and the function with respect to time are allowed in the nonlinear terms. Compared with the assumption about the nonlinear terms in $[19,20]$, the assumption in this paper is more general. Thus, a time-varying gain design method is introduced to achieve the control goals of the concerned system.

\section{Preliminaries}

In this paper, we consider a class of nonlinear systems in the following form: 


$$
\left\{\begin{array}{l}
\dot{\xi}_{1}(t)=\xi_{2}(t)+\psi_{1}(t, \xi(t), u(t)), \\
\dot{\xi}_{2}(t)=\xi_{3}(t)+\psi_{2}(t, \xi(t), u(t)), \\
\vdots \\
\dot{\xi}_{n-1}(t)=\xi_{n}(t)+\psi_{n-1}(t, \xi(t), u(t)), \\
\dot{\xi}_{n}(t)=u(t)
\end{array}\right.
$$

where $\xi(t)=\left[\xi_{1}(t), \xi_{2}(t), \ldots, \xi_{n}(t)\right]^{T} \in \mathbb{R}^{n}$ is the state and $u(t) \in \mathbb{R}$ is the input; the uncertain continuous functions $\psi_{i}(t, \xi(t), u(t)): \mathbb{R}^{+} \times \mathbb{R}^{n} \times \mathbb{R} \longrightarrow \mathbb{R}, \quad i=1,2, \ldots, n-1$, satisfy the following growth condition.

Assumption 1. For all $(t, \xi(t), u(t)) \in \mathbb{R}^{+} \times \mathbb{R}^{n} \times \mathbb{R}$, the following inequalities hold:

$$
\begin{aligned}
\left|\psi_{i}(t, \xi(t), u(t))\right| \leq & \theta(1+t)^{c}\left(\left|\xi_{i+2}(t)\right|+\left|\xi_{i+3}(t)\right|+\cdots\right. \\
& \left.+\left|\xi_{n}(t)\right|+|u(t)|\right), \quad i=1,2, \ldots, n-1,
\end{aligned}
$$

where $\theta$ is an unknown positive constant and $c$ is a known constant which satisfies $0 \leq c<1$.

Remark 1. Assumption 1 is a reasonable condition. According to Assumption 1, we can know that the nonlinear terms of system (1) can include the unknown constant and the function with respect to time. Therefore, compared with the assumption in $[19,20]$, Assumption 1 is more general, and the nonlinear characteristics of system (1) are more diverse. The controller designed in this paper is effective for a class of nonlinear systems as long as the nonlinear terms satisfy (2).

The control goal of this paper is to construct a state feedback controller such that all the signals of the closedloop system converge to zero. As long as the context does not cause confusion, the parameters of the function can be simplified.

\section{Main Results}

Theorem 1. When the constant $c$ satisfies $0<c<0.5$, all the signals of system (1) can converge to zero by the following controller:

$$
u=-\frac{\beta_{1}}{(t+1)^{\gamma n}} \xi_{1}-\frac{\beta_{2}}{(t+1)^{\gamma(n-1)}} \xi_{2}-\cdots-\frac{\beta_{n}}{(t+1)^{\gamma}} \xi_{n},
$$

where $\beta_{i}, i=1,2, \ldots, n$, are coefficients of the Hurwitz polynomial $\phi(\rho)=\rho^{n}+\beta_{n} \rho^{n-1}+\cdots+\beta_{2} \rho+\beta_{1}$ and $\gamma=2 c$.

Proof. Let $\gamma=2 c$. One presents the coordinate transformations as follows:

$$
\epsilon_{i}=(t+1)^{\gamma i} \xi_{i}, \quad i=1,2, \ldots, n .
$$

Based on (1) and (4), it is obtained that

$$
\dot{\epsilon}_{i}=\frac{1}{(t+1)^{\gamma}} \epsilon_{i+1}+(t+1)^{\gamma i} \psi_{i}+\frac{\gamma i}{t+1} \epsilon_{i}, \quad i=1,2, \ldots, n \text {, }
$$

where $\epsilon_{n+1}=u$.
Letting

$$
u=-\frac{1}{(t+1)^{\gamma(n+1)}}\left(\beta_{1} \epsilon_{1}+\beta_{2} \epsilon_{2}+\cdots+\beta_{n} \epsilon_{n}\right),
$$

where $\beta_{i}, i=1,2, \ldots, n$, are given in (3), the following equation is satisfied:

$$
\dot{\epsilon}=\frac{1}{(t+1)^{\gamma}} \Phi \epsilon+\frac{\gamma}{t+1} \Lambda \epsilon+\Psi
$$

where $\epsilon=\left[\epsilon_{1}, \epsilon_{2}, \ldots, \epsilon_{n}\right]^{T}, \Lambda=\operatorname{diag}[1,2, \ldots, n]$, and

$$
\begin{aligned}
& \Phi=\left[\begin{array}{cccc}
0 & 1 & \cdots & 0 \\
\vdots & \vdots & \ddots & 0 \\
0 & 0 & \cdots & 1 \\
-\beta_{1} & -\beta_{2} & \cdots & -\beta_{n}
\end{array}\right], \\
& \Psi=\left[\begin{array}{c}
(t+1)^{\gamma} \psi_{1} \\
\vdots \\
(t+1)^{(n-1) \gamma} \psi_{n-1} \\
0
\end{array}\right] .
\end{aligned}
$$

Because $\beta_{i}, i=1,2, \ldots, n$, are coefficients of the Hurwitz polynomial $\phi(\rho)$, there is a positive definite matrix $\Gamma$ satisfying $\Gamma \Phi+\Phi^{T} \Gamma \leq-I$ [21]. Letting $V_{\epsilon}=\epsilon^{T} \Gamma \epsilon$, one gets

$$
\left.\dot{V}_{\epsilon}\right|_{(7)} \leq-\frac{1}{(t+1)^{\gamma}}\|\epsilon\|^{2}+\frac{2 \gamma\|\Lambda \Gamma\|}{t+1}\|\epsilon\|^{2}+2 \epsilon^{T} \Gamma \Psi \text {. }
$$

By Assumption 1 and (4), one has

$$
\begin{aligned}
\left|(t+1)^{i \gamma} \psi_{i}(t, \xi, u)\right| & \leq \frac{\theta(1+\delta)}{(t+1)^{1.5 \gamma}}\left(\left|\epsilon_{1}\right|+\left|\epsilon_{2}\right|+\cdots+\left|\epsilon_{n}\right|\right) \\
& \leq \frac{\sqrt{n} \theta(1+\delta)}{(t+1)^{1.5 \gamma}}\|\epsilon\| .
\end{aligned}
$$

It follows from (10) that

$2 \epsilon^{T} \Gamma \Psi \leq 2\|\epsilon\| \cdot\|\Gamma\| \cdot\|\Psi\| \leq \frac{2\|\Gamma\| \sqrt{n(n-1)} \theta(1+\delta)}{(t+1)^{1.5 \gamma}}\|\epsilon\|^{2}$.

Substituting (11) into (9), one gets

$$
\begin{aligned}
\left.\dot{V}_{\epsilon}\right|_{(7)} \leq & -\frac{1}{(t+1)^{\gamma}}\|\epsilon\|^{2}+\frac{2 \gamma\|\Lambda \Gamma\|}{t+1}\|\epsilon\|^{2} \\
& +\frac{2\|\Gamma\| \sqrt{n(n-1)} \theta(1+\delta)}{(t+1)^{1.5 \gamma}}\|\epsilon\|^{2} .
\end{aligned}
$$

Since $\gamma=2 c<1$, there is a finite time $T$ such that

$$
-\frac{1}{(t+1)^{\gamma}}+\frac{2 \gamma\|\Lambda \Gamma\|}{t+1}+\frac{2\|\Gamma\| \sqrt{n(n-1)} \theta(1+\delta)}{(t+1)^{1.5 \gamma}}<0, \quad t \geq T \text {. }
$$

Therefore, it is verified from (12) that

$$
\dot{V}<-\|\epsilon\|^{2}, \quad \text { for } t \geq T \text {. }
$$

Based on (14) and the definition of $V$, the states $\epsilon_{i}$, $i=1,2, \ldots, n$, converge to zero. By (6), the controller $u$ 
converges to zero. From (4), one has that the states $\xi_{i}$, $i=1,2, \ldots, n$, converge to zero.

Remark 2. From the proof of Theorem 1, we can see that the constant $c$ is a key design parameter. As long as the parameter $c$ satisfies the condition $0<c<0.5$, one guarantees that equation (13) holds, and then, one handles the effects of the unknown parameter $\theta$ and the function on time in the nonlinear terms. The parameters in controller (3) consist of two parts. One is the parameter $\gamma$, which only needs to be satisfied by $\gamma=2 c$. The other part is the Hurwitz polynomial coefficients $\beta_{i}, i=1,2, \ldots, n$, which are also relatively easy to choose. Therefore, the parameters in controller (3) are better selected, which avoid excessive calculation process.

Theorem 2. In the case of $c=0$, the states of system (1) can converge to zero by the following controller:

$$
u=-\frac{\beta_{1} \xi_{1}}{(t+1)^{0.5 n}}-\frac{\beta_{2} \xi_{2}}{(t+1)^{0.5(n-1)}}-\cdots-\frac{\beta_{n} \xi_{n}}{(t+1)^{0.5}},
$$

where $\beta_{i}, i=1,2, \ldots, n$, are coefficients of the Hurwitz polynomial $\phi(\rho)=\rho^{n}+\beta_{n} \rho^{n-1}+\cdots+\beta_{2} \rho+\beta_{1}$.

Proof. The proof procedure is similar to the proof procedure of Theorem 1 . One chooses $\gamma=0.5$ in (4), and then, controller (15) is designed. In order to avert repetition, the detailed proof is omitted.

Theorem 3. When the constant $c$ satisfies $0.5 \leq c<1$, all the signals of system (1) can converge to zero by the following controller:

$$
u=-\frac{\beta_{1}}{(t+1)^{n}} \xi_{1}-\frac{\beta_{2}}{(t+1)^{n-1}} \xi_{2}-\cdots-\frac{\beta_{n}}{t+1} \xi_{n},
$$

where $\beta_{i}, i=1,2, \ldots, n$, are coefficients of the Hurwitz polynomial $\phi(\rho)=\rho^{n}+\beta_{n} \rho^{n-1}+\cdots+\beta_{2} \rho+\beta_{1}$.

Proof. One presents the coordinate transformations as follows:

$$
\epsilon_{i}=(t+1)^{i} \xi_{i}, \quad i=1,2, \ldots, n .
$$

Based on (16) and (17), it is obtained that

$$
\dot{\epsilon}_{i}=\frac{1}{t+1} \epsilon_{i+1}+(t+1)^{i} \psi_{i}+\frac{i}{t+1} \epsilon_{i}, \quad i=1,2, \ldots, n .
$$

Letting

$$
u=-\frac{1}{(t+1)^{n+1}}\left(\beta_{1} \epsilon_{1}+\beta_{2} \epsilon_{2}+\cdots+\beta_{n} \epsilon_{n}\right),
$$

where $\beta_{i}, i=1,2, \ldots, n$, are given in (3), the following equation is satisfied:

$$
\dot{\epsilon}=\frac{1}{t+1} \Phi \epsilon+\frac{1}{t+1} \Lambda \epsilon+\Psi
$$

where $\epsilon=\left[\epsilon_{1}, \epsilon_{2}, \ldots, \epsilon_{n}\right]^{T}, \Lambda=\operatorname{diag}[1,2, \ldots, n]$, and

$$
\begin{aligned}
& \Phi=\left[\begin{array}{cccc}
0 & 1 & \cdots & 0 \\
\vdots & \vdots & \ddots & 0 \\
0 & 0 & \cdots & 1 \\
-\beta_{1} & -\beta_{2} & \cdots & -\beta_{n}
\end{array}\right], \\
& \Psi=\left[\begin{array}{c}
(t+1) \psi_{1} \\
\vdots \\
(t+1)^{n-1} \psi_{n-1} \\
0
\end{array}\right] .
\end{aligned}
$$

Because $\beta_{i}, i=1,2, \ldots, n$, are coefficients of the Hurwitz polynomial $\phi(\rho)$, there is a positive definite matrix $\Gamma$ satisfying $\Gamma(\Phi+\Lambda)+(\Phi+\Lambda)^{T} \Gamma \leq-I[21]$. Letting $V_{\epsilon}=\epsilon^{T} \Gamma \epsilon$, one gets

$$
\left.\dot{V}_{\epsilon}\right|_{(7)} \leq-\frac{1}{t+1}\|\epsilon\|^{2}+2 \epsilon^{T} \Gamma \Psi .
$$

By Assumption 1 and (4), one has

$$
\begin{aligned}
\left|(t+1)^{i} \psi_{i}(t, \xi, u)\right| & \leq \frac{\theta(1+\delta)}{(t+1)^{2-c}}\left(\left|\epsilon_{1}\right|+\left|\epsilon_{2}\right|+\cdots+\left|\epsilon_{n}\right|\right) \\
& \leq \frac{\sqrt{n} \theta(1+\delta)}{(t+1)^{2-c}}\|\epsilon\| .
\end{aligned}
$$

It follows from (10) that

$2 \epsilon^{T} \Gamma \Psi \leq 2\|\epsilon\| \cdot\|\Gamma\| \cdot\|\Psi\| \leq \frac{2\|\Gamma\| \sqrt{n(n-1)} \theta(1+\delta)}{(t+1)^{2-c}}\|\epsilon\|^{2}$.

Substituting (11) into (9), one gets

$\left.\dot{V}_{\epsilon}\right|_{(7)} \leq-\frac{1}{t+1}\|\epsilon\|^{2}+\frac{2\|\Gamma\| \sqrt{n(n-1)} \theta(1+\delta)}{(t+1)^{2-c}}\|\epsilon\|^{2}$.

Since $c<1$, there is a finite time $T$ such that

$$
-\frac{1}{t+1}+\frac{2\|\Gamma\| \sqrt{n(n-1)} \theta(1+\delta)}{(t+1)^{2-c}}<0, \quad t \geq T .
$$

Therefore, it is verified from (12) that

$$
\dot{V}<-\|\epsilon\|^{2}, \quad \text { for } t \geq T \text {. }
$$

Based on (27) and the definition of $V$, the states $\epsilon_{i}$, $i=1,2, \ldots, n$, converge to zero. By (20), the controller $u$ converges to zero. From (17), one has that the states $\xi_{i}$, $i=1,2, \ldots, n$, converge to zero.

Remark 3. In this paper, with the help of the Lyapunov function, a new control strategy is proposed for upper triangular nonlinear systems, and state feedback controllers (3), (15), and (16) are designed such that all the signals of the closed-loop system converge to zero. In Theorem 2, when $c=0$, one can choose $\gamma=0.5$. Then, controller (15) can ensure the convergence performance of the states. In fact, when $c=0$, as long as the constant $\gamma<1$ is selected, the effectiveness of controllers (15) and (16) can be ensured. 


\section{Simulation Examples}

Example 1. One considers the following nonlinear system:

$$
\left\{\begin{array}{l}
\dot{\xi}_{1}=\xi_{2}+0.1(1+t)^{0.2}\left(\xi_{3}+u\right), \\
\dot{\xi}_{2}=\xi_{3}+0.1(1+t)^{0.2} u, \\
\dot{\xi}_{n}=u
\end{array}\right.
$$

One gets that system (28) satisfies Assumption 1 with $\theta=0.1$ and $c=0.2$. Let $\gamma=0.4, \beta_{1}=0.3, \beta_{2}=1.2$, and $\beta_{3}=0.7$. Based on Theorem 1, a state feedback controller for system (28) is designed as

$$
u=-\frac{3 \xi_{1}}{10(t+1)^{1.2}}-\frac{6 \xi_{2}}{5(t+1)^{0.8}}-\frac{7 \xi_{3}}{10(t+1)^{0.4}} .
$$

The initial condition is chosen as $\xi_{1}(0)=0.5$, $\xi_{2}(0)=0.8$, and $\xi_{3}(0)=0.8$. From Figures $1-3$, one has that the states $\xi_{1}, \xi_{2}$, and $\xi_{3}$ of system (28) converge to zero. The controller $u$ is shown in Figure 4 .

Example 2. A practical example about the resonant circuit system is investigated as follows [22]

$$
\left\{\begin{array}{l}
\dot{i}_{\eta_{1}}=-\frac{v_{\tau}}{\eta_{1}}-\frac{r_{1}}{\eta_{1}} i_{\eta_{2}}+\frac{r_{1}}{2 \eta_{1}} \sin v_{\tau}, \\
\dot{v}_{\tau}=\frac{i_{\eta_{2}}}{\tau}-\frac{1}{2 \tau} \sin v_{\tau}, \\
\dot{i}_{\eta_{2}}=-\frac{r_{2}}{\eta_{2}} i_{\eta_{2}}+\frac{\mu}{\eta_{2}} .
\end{array}\right.
$$

In system (30), the meaning of the parameters is found in [22]. Following the coordinate transformation in [22], one gets

$$
\left\{\begin{array}{l}
\dot{\xi}_{1}=\xi_{2}+2 \xi_{3} \\
\dot{\xi}_{2}=\xi_{3} \\
\dot{\xi}_{n}=u
\end{array}\right.
$$

One gets that system (30) satisfies Assumption 1 with $\theta=2$ and $c=0$. Let $\gamma=0.5, \beta_{1}=0.3, \beta_{2}=1.2$, and $\beta_{3}=0.7$. Based on Theorem 2, a state feedback controller for system (30) is designed as

$$
u(t)=-\frac{\beta_{1} \xi_{1}}{(t+1)^{1.5}}-\frac{\beta_{2} \xi_{2}}{(t+1)}-\frac{\beta_{3} \xi_{3}}{(t+1)^{0.5}} .
$$

The initial condition is chosen as $\xi_{1}(0)=-0.5$, $\xi_{2}(0)=-0.3$, and $\xi_{3}(0)=-0.5$. From Figures $5-7$, one has that the states $\xi_{1}, \xi_{2}$, and $\xi_{3}$ of system (30) converge to zero. The controller $u$ is shown in Figure 8 .

Example 3. One considers the following nonlinear system:

$$
\left\{\begin{array}{l}
\dot{\xi}_{1}=\xi_{2}+0.5(1+t)^{0.9}\left(\xi_{3}+u\right), \\
\dot{\xi}_{2}=\xi_{3}+0.5(1+t)^{0.9} u, \\
\dot{\xi}_{n}=u .
\end{array}\right.
$$

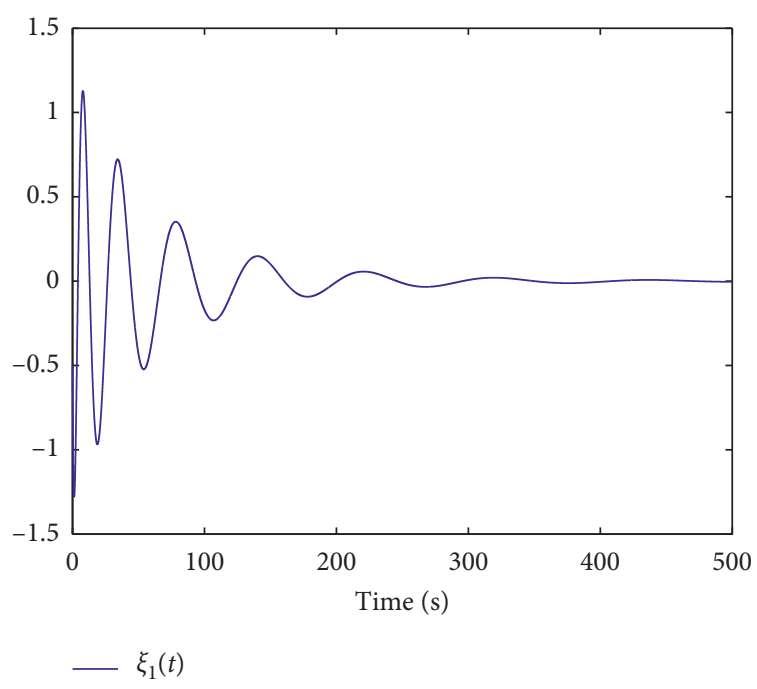

FIgURE 1: Trajectory of $\xi_{1}(t)$.

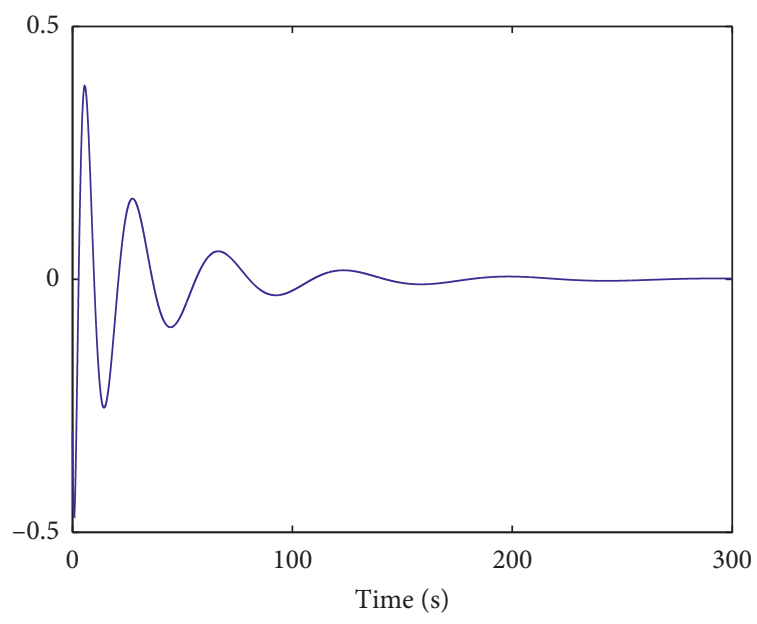

$-\xi_{2}(t)$

Figure 2: Trajectory of $\xi_{2}(t)$.

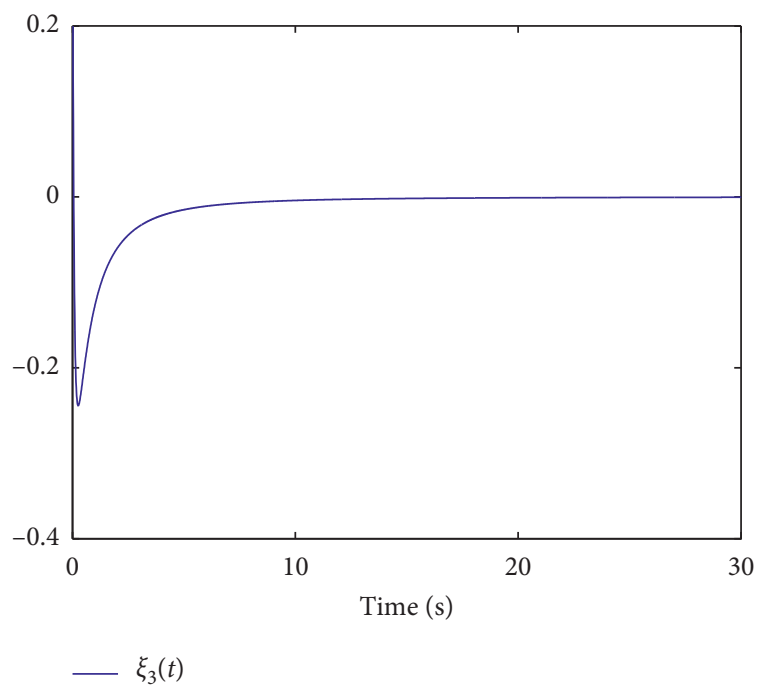

FIgURE 3: Trajectory of $\xi_{3}(t)$. 


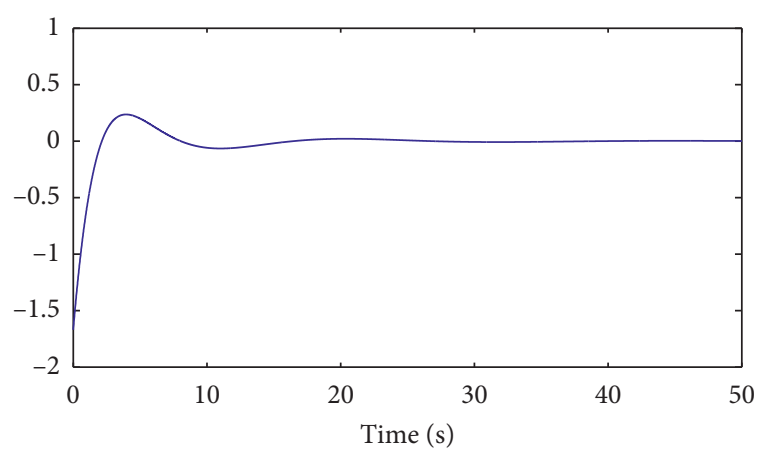

$-u(t)$

Figure 4: Trajectory of $u(t)$.

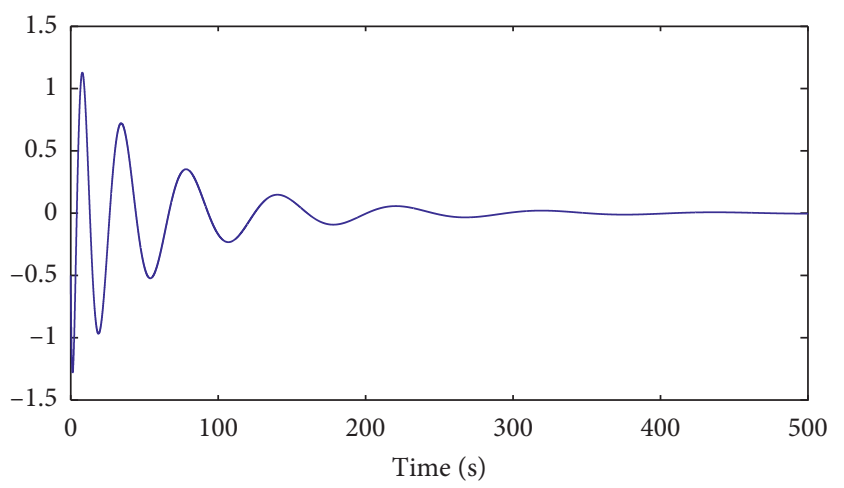

Figure 5: Trajectory of $\xi_{1}(t)$

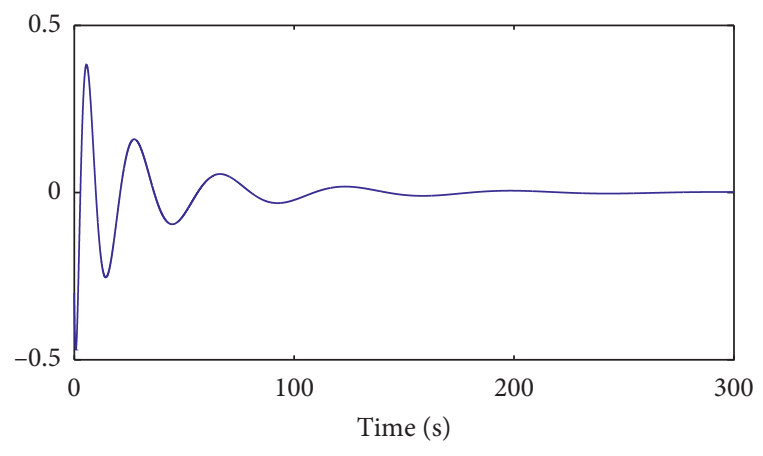

$\xi_{2}(t)$

Figure 6: Trajectory of $\xi_{2}(t)$.

One gets that system (33) satisfies Assumption 1 with $\theta=0.5$ and $c=0.9$. Let $\beta_{1}=5, \beta_{2}=10$, and $\beta_{3}=5$. Based on Theorem 1, a state feedback controller for system (33) is designed as

$$
u=-\frac{5 \xi_{1}}{(t+1)^{3}}-\frac{10 \xi_{2}}{(t+1)^{2}}-\frac{5 \xi_{3}}{(t+1)}
$$

The initial condition is chosen as $\xi_{1}(0)=0.4$, $\xi_{2}(0)=0.4$, and $\xi_{3}(0)=0.3$. From Figures 9-11, one has that the states $\xi_{1}, \xi_{2}$, and $\xi_{3}$ of system (33) converge to zero. The controller $u$ is shown in Figure 12 .

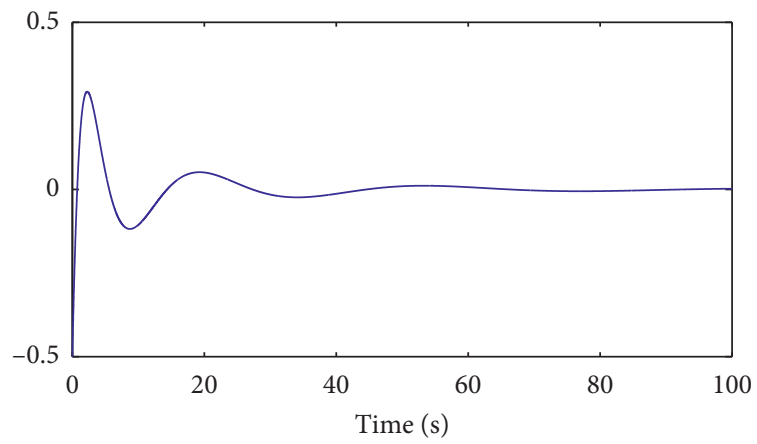

$\xi_{3}(t)$

Figure 7: Trajectory of $\xi_{3}(t)$.

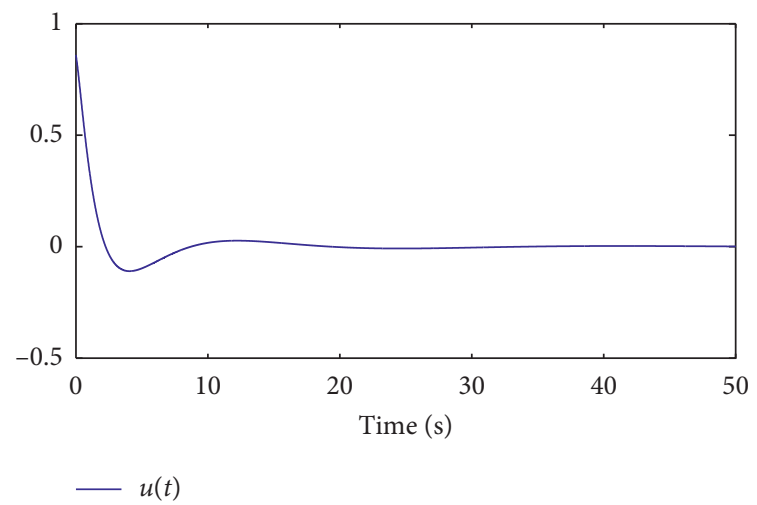

Figure 8: Trajectory of $u(t)$.

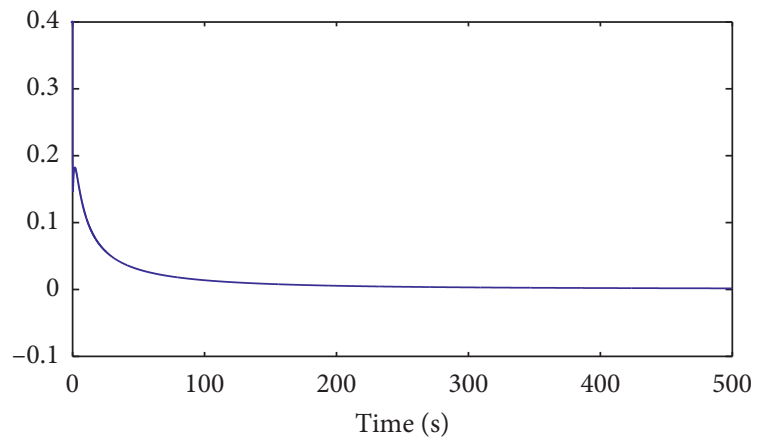

$\xi_{1}(t)$

Figure 9: Trajectory of $\xi_{1}(t)$.

Remark 4. In the two simulation examples, one can see that the parameters in controllers (29), (32), and (34) are relatively easy to select, which do not require a large amount of calculation to deal with the unknown parameter in systems (28), (30), and (33), respectively. In addition, controllers (29), (32), and (34) are effective for a class of nonlinear systems as long as the nonlinear terms satisfy (2). Compared with the example of the results $[18,22]$, the unknown constant and the function with respect to time are allowed in the nonlinear terms. Compared with the commonly adaptive control strategy, the time-varying gain design method is 


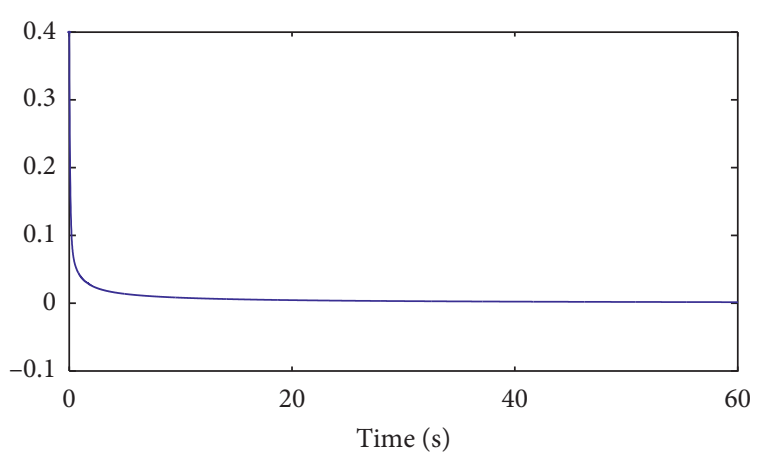

$-\xi_{2}(t)$

Figure 10: Trajectory of $\xi_{2}(t)$.

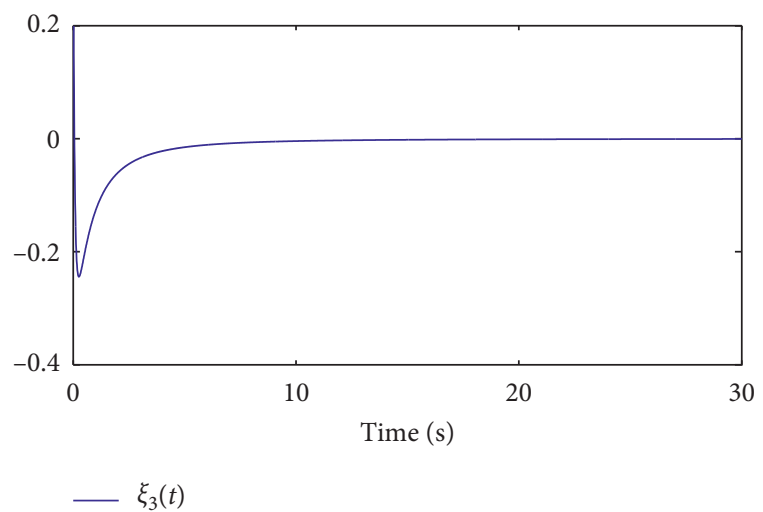

Figure 11: Trajectory of $\xi_{3}(t)$.

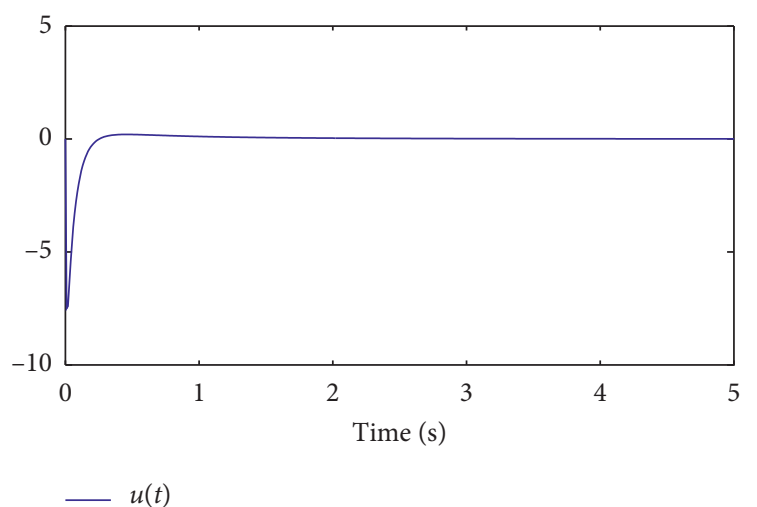

Figure 12: Trajectory of $u(t)$.

more concise, the calculation process is less, and a lot of calculation work is reduced.

\section{Conclusion}

This paper has investigated the state feedback control problem of upper triangular nonlinear systems. One has assumed that the nonlinear term recognizes an incremental rate relying on the unknown constant and the function with respect to time. A time-varying gain design method has been used to construct a state feedback controller. With the help of a Lyapunov function, one has obtained that all the signals of the closed-loop system have converged to zero. Finally, two numerical examples have been presented to illustrate the effectiveness of the time-varying gain design method.

\section{Data Availability}

All figures are made by Matlab.

\section{Conflicts of Interest}

The author declares no conflicts of interest.

\section{Acknowledgments}

This work was supported by the National Natural Science Foundation of China (nos. 61503214 and 61873334).

\section{References}

[1] M. Krstic, I. Kanellakopoulos, and P. V. Kokotovic, Nonlinear and Adaptive Control Design, Wiley, New York, NY, USA, 1995.

[2] A. M. Bloch, Nonholonomic Mechanics and Control, SpringerVerlag, New York, NY, USA, 2003.

[3] K. Liu, G. Xie, W. Ren, and L. Wang, "Consensus for multiagent systems with inherent nonlinear dynamics under directed topologies," Systems \& Control Letters, vol. 62, no. 2, pp. 152-162, 2013.

[4] R. Guo, "Projective synchronization of a class of chaotic systems by dynamic feedback control method," Nonlinear Dynamics, vol. 90, no. 1, pp. 53-64, 2017.

[5] H. Li, X. Zhang, and Q. Liu, "Adaptive output feedback control for a class of large-scale output-constrained nonlinear time-delay systems," IET Control Theory \& Applications, vol. 12, no. 1, pp. 174-181, 2018.

[6] K. Liu and Z. Ji, "Consensus of multi-agent systems with time delay based on periodic sample and event hybrid control," Neurocomputing, vol. 270, no. 27, pp. 11-17, 2017.

[7] K. Liu, Z. Ji, and X. Zhang, "Periodic event-triggered consensus of multi-agent systems under directed topology," Neurocomputing, vol. 385, no. 14, pp. 33-41, 2020.

[8] R. Xu and F. Zhang, ". $\epsilon$-nash mean-field games for general linear-quadratic systems with applications," Automatica, vol. 114, pp. 1-6, 2020.

[9] X. Lu and H. Li, "An improved stability theorem for nonlinear systems on time scales with application to multi-agent systems," IEEE Transactions on Circuits and Systems II: Express Briefs, 2020.

[10] X. Yi, R. Guo, and Y. Qi, "Stabilization of chaotic systems with both uncertainty and disturbance by the UDE-based control method," IEEE Access, vol. 8, no. 1, pp. 62471-62477, 2020.

[11] C. Qian and J. Li, "Global finite-time stabilization by output feedback for planar systems without observable linearization," IEEE Transactions on Automatic Control, vol. 50, no. 6, pp. 885-890, 2005.

[12] H. Li, X. Zhang, and G. Feng, "Event-triggered output feedback control of switched nonlinear systems with input saturation," IEEE Transactions on Cybernetics, 2020.

[13] C. Hua, X. Guan, and P. Shi, "Robust backstepping control for a class of time delayed systems," IEEE Transactions on Automatic Control, vol. 50, no. 6, pp. 894-899, 2005. 
[14] X. Zhang, L. Liu, and G. Feng, "Leader-follower consensus of time-varying nonlinear multi-agent systems," Automatica, vol. 52, no. 2, pp. 8-14, 2015.

[15] X. Zhang, L. Baron, Q. Liu, and E.-K. Boukas, "Design of stabilizing controllers with a dynamic gain for feedforward nonlinear time-delay systems," IEEE Transactions on Automatic Control, vol. 56, no. 3, pp. 692-697, 2011.

[16] B. Niu and L. Li, "Adaptive backstepping-based neural tracking control for MIMO nonlinear switched systems subject to input delays," IEEE Transactions on Neural Networks and Learning Systems, vol. 29, no. 6, pp. 2638-2644, 2018.

[17] X. Ye, J. Huang, and H. Unbehauen, "Decentralized robust stabilization for large-scale feedforward non-linear systems," International Journal of Control, vol. 79, no. 12, pp. 1505-1511, 2006.

[18] H. Li, X. Zhang, and L. Chang, "Output feedback regulation of a class of triangular structural nonlinear systems with unknown measurement sensitivity," International Journal of Systems Science, vol. 50, no. 13, pp. 2486-2496, 2019.

[19] L. Chang, C. Zhang, X. Zhang, and X. Chen, "Decentralised regulation of nonlinear multi-agent systems with directed network topologies," International Journal of Control, vol. 90, no. 11, pp. 2338-2348, 2017.

[20] H. Li, X. Zhang, Z.-M. Wang, and A. Wei, "Global output feedback stabilisation of a class of stochastic systems with unknown growth rate," International Journal of Control, 2019.

[21] L. Praly and Z. P. Jiang, "Linear output feedback with dynamic high gain for nonlinear systems," Systems \& Control Letters, vol. 53, no. 2, pp. 107-116, 2004.

[22] H. Li, X. Zhang, and M. Li, "Design of output feedback controller for stochastic feedforward systems with unknown measurement sensitivity," ISA Transactions, vol. 97, pp. 182-188, 2020. 